\title{
Bistatic Cross Section of Ships between P and S Band
}

\author{
Andrew Stove, Michail Antoniou, Dimitrios Tzagkas \\ Department of Electrical, Electronic and Systems \\ Engineering \\ University of Birmingham \\ Birmingham, U.K
}

stovea@bham.ac.uk

\author{
Matthew Ritchie \\ Radar Sensing Team, \\ Defence Science and Technology Laboratory, Porton Down, \\ Wilts, SP4 0JQ \\ UK
}

\begin{abstract}
This paper presents some data on the bistatic radar cross sections of ships at Ultra High Frequencies and at lower microwave frequencies. Although the quantity of data is limited, it is much more than has been published before. It allows a rule of thumb to be deduced: that the bistatic radar cross section is approximately the square root of its monostatic value when both values are measured in square metres.
\end{abstract}

Keywords—bistatic; radar cross section; ship

\section{INTRODUCTION}

There is a growing interest in bistatic radars because, primarily, of the following advantages:

a) Operational 'stealth', in that the receiver site ideally emits no radiation,

b) the use of someone else's transmissions avoids the cost and weight of a dedicated transmitter

c) the geometry allows the target to be seen from different aspects

d) more favourable clutter statistics in comparison to monostatic radar [1, 2. 3] and

e) the use of an existing transmitter as an illuminator allows the radar to operate in frequency bands for which an dedicated frequency allocation would not be obtainable. 5]

Overviews of the field of bistatic radar can be found in [4,

One of the potential fields of application of bistatic radar is in marine radars $[6,7]$ and at least one significant campaign has been undertaken to gather bistatic sea clutter data $[1,2,3$, $8]$, but very little has been published on the bistatic radar cross sections of ships.

The preponderance of clutter data is necessary because radars must typically, indeed almost universally, operate at low false alarm rates so the 'tails' of the clutter distribution must be characterized, whereas the detection requirements are more modest and can be estimated from the values nearer the mode of the distribution, so much less data is required. Some experimental data is, however, required.
Each measurement campaign typically yields only one or two measurements on ships. When many of these measurements are analysed, as will be seen below, they seem anomalously low. When, however, even a relatively small number or results are compared, they show a similar trend so that we can begin to make at least provisional statements about the bistatic radar cross section of ships. The limited quantity of data means that what is deduced is more of a 'rule of thumb' than a 'model,' but it is still useful in the absence of a more detailed model and will be of value in validating the results of electromagnetic modeling.

\section{RADAR DATA}

As mentioned in the introduction, the data set is small, comprising 11 data points. The ships which were measured were a harbour-master's tender, a naval tender, three warships, two ferries and a large cargo ship. More than one data point was available on some of the ships.

The measurements were made using different radar systems at frequencies between approximately $700 \mathrm{MHz}$ and $2.4 \mathrm{GHz}$ so extrapolation to, for example, X-band must be made with caution. The S-band data was generated using the University College London (UCL) NetRad system in trials described within [8]. Only one of the L-band values has been reported before, within [6]. The $700 \mathrm{MHz}$ results were obtained as part of the work described in [7].

The measurements were also made over a number of different out of plane bistatic angles from about $10^{\circ}$ to $150^{\circ}$ in azimuth and $0^{\circ}$ to $40^{\circ}$ in elevation

Although it could be considered a gross simplification to average the data across such a range of frequencies and, particularly, over such a range of bistatic angles, it was judged that the small quantity of data did not justify refining the data categories.

None of the ships measured would be considered to be particularly 'stealthy,' i. e. no very extreme steps had been taken to minimize their monostatic radar cross sections (in which case it might be expected that the bistatic radar cross section might be larger than the 'stealthed' monostatic radar cross section). 


\section{EQuivalent MonOSTATIC RADAR CROSS SECTION}

In order to produce at least a 'rule of thumb' estimating the bistatic radar cross section of ships, some compensation must be made for the size of the ship. The analysis presented here therefore compares the measured values of the bistatic radar cross section with the monostatic radar cross section.

In a few cases the monostatic radar cross section was measured at the same time as the bistatic radar cross section, allowing a direct comparison to be made. For the cases where the monostatic radar cross section was not measured simultaneously, it was estimated using the rule of thumb that the radar cross section in square meters is equal to the displacement, $D$, in tons [9], i.e.:

$$
\sigma_{\text {mono }}=D
$$

Reference [10] suggests higher values are applicable at low grazing angles:

$$
\sigma_{\text {mono }}=0.52 D_{k}^{1 / 2} f_{M}^{3 / 2}
$$

where $D_{k}$ is the displacement in kilo-tonnes and $f_{M}$ is the frequency in $\mathrm{MHz}$, but many measurements suggest that (1) is a better approximation even at grazing incidence. In particular, analysis of the data in table 8 of reference [11] shows that the data follows equation (1) almost perfectly, although it should be noted that this specific data refers to X-band rather than to the frequencies at which the bistatic data discussed here were measured.

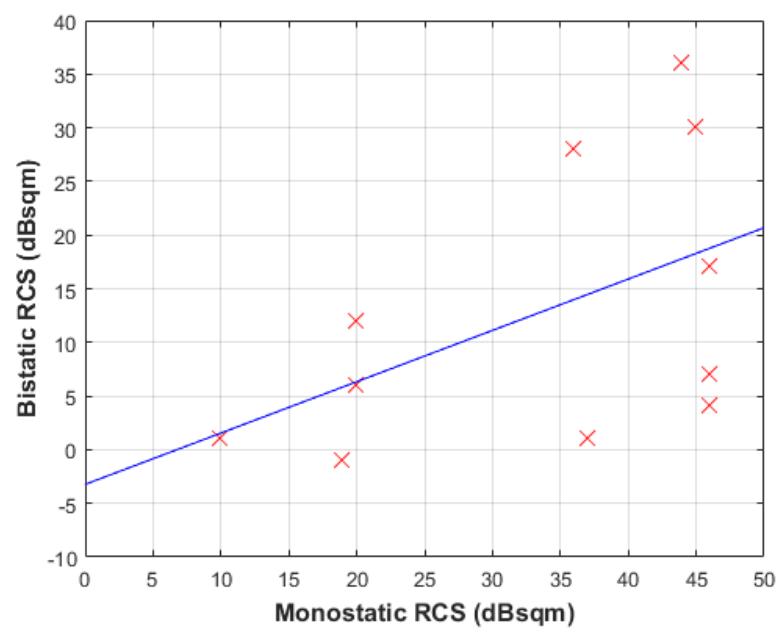

Figure 1: Bistatic v. Monostatic Radar Cross Sections of Ships

Figure 1 shows the data points. It had been suspected and as has been assumed before by many authors, that the bistatic radar cross sections are substantially less than monostatic values. This figure includes data gathered from multiple geometries and different targets, which is a simplification of the potentally multi-dimensional problem, but the overall trend does still show that the bistatic radar cross section is generally lower than the monostatic.
The figure also shows a straight-line fit to the data points. It follows the line:

$$
\sigma_{b i}=0.495 \sigma_{\text {mono }}-3.27
$$

where $\sigma_{b i}$ is the bistatic radar cross section and the radar cross sections are measured in $\mathrm{dBm}^{2}$. It should be noted, however, that the regression coefficient was only 0.5 so the reliability of the curve is limited, and certainly the values are not meaningful beyond the first significant digit. There is no reason to believe that true law is in fact a straight line, but this is the simplest plausible curve and there is insufficient data to make it worth considering a more sophisticated fit.

Figures 2 and 3 show the histogram of deviations from the best-fit straight line. Figure 2 shows all the data points and Figure 3 shows those data points which are reasonably close to the fitted line.

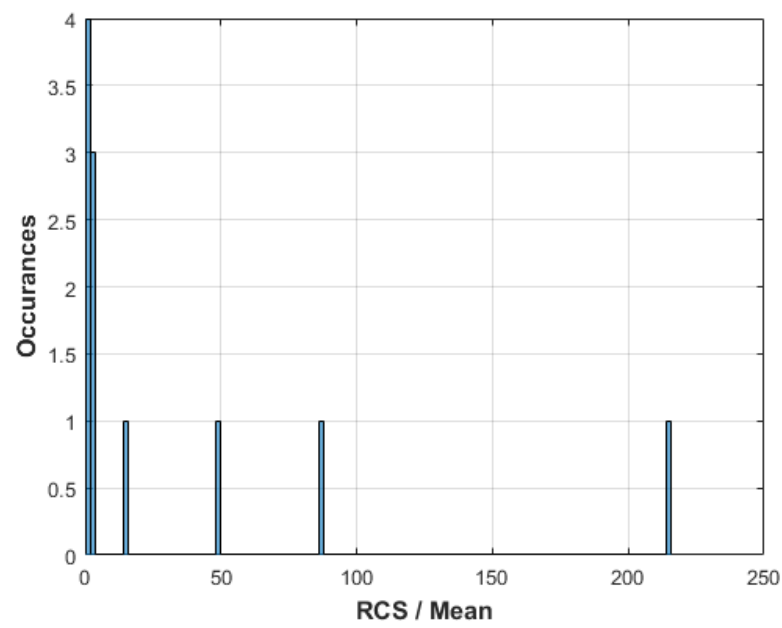

Figure 2: Deviation of Observed Bistatic Radar Cross Sections of Ships from Best-Fit - Outliers

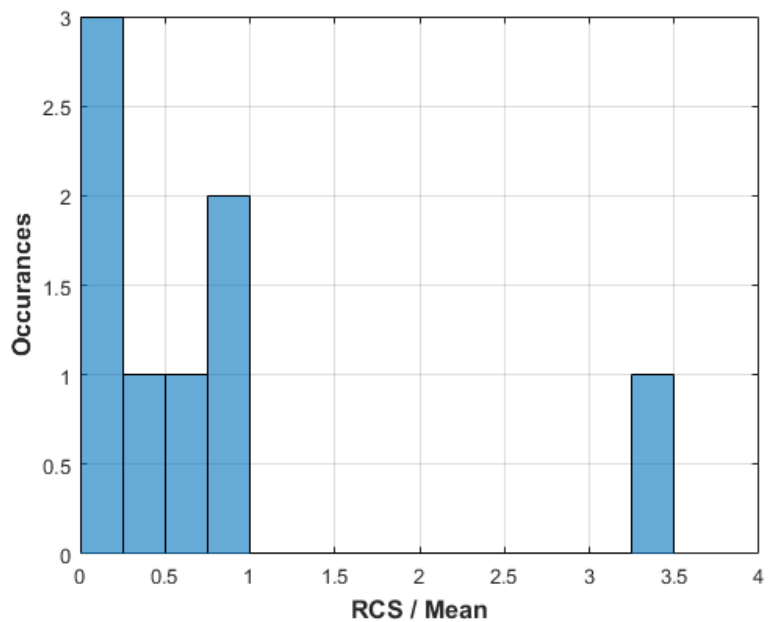

Figure 3: Deviation of Observed Bistatic Radar Cross Sections of Ships from Best-Fit - Minor Deviations

Both figures show that the probability density is highest for an abscissa of zero. This is what would be expected from a 
Swerling-1 or Swerling-2 distribution [12] so we can expect the individual radar cross section measurements to follow such a distribution around a mean value predicted by the fit, rather than another distribution, such as Swerling-3 or Swerling-4 [12] which has a density of zero for a value of zero

Figure 2 does show that some relatively large bistatic radar cross sections have been seen, but none are greater than the expected monostatic value. These may be considered as corresponding to the 'flashes' which occur in the monostatic radar cross section.

Figure 3 indicates that for low of the cross section the 'fit' to the exponential distribution is plausible. Since the minimum value of the radar cross section is generally what needs to be known in order to prove that a radar will be able to perform a particular task, it is the data in Figure 3 which is probably more relevant,

The available data can be used to support the following statements:

a) The mean bistatic radar cross section of a ship is related to the expected monostatic radar cross section by equation (1).

b) The minimum bistatic radar cross section can be assumed to be related to the mean value by an exponential (Swerling case 1 or 2 distribution).

c) The bistatic radar cross section also exhibits much higher radar cross sections in some directions

d) The maximum value of the bistatic radar cross section is equal to the expected monostatic radar cross section.

The residual errors were analysed for any dependence on frequency or aspect angle. The fit with frequency was of the form

$$
\sigma_{b i}=0.495 \sigma_{\text {mono }}-0.18-1.63 f
$$

Where $f$ is the frequency in $\mathrm{dBGHz}$ (i. e. $\log _{10}\left(f^{\prime}\right)$ where $f^{\prime}$ is the frequency in $\mathrm{GHz}$ ). The correlation coefficient for the dependence with frequency was, however, only 0.27 so this result is very unreliable.

The limited data available showed no variation with aspect angle.

\section{DISSCUSSION}

A well-known way to estimate the bistatic cross section which has previously been suggested by Crispin et al. [13] is the so-called monostatic-bistatic equivalence theorem. This states that the bistatic radar cross section is equal to the monostatic radar cross section at the mid-point between the direction at which the transmitter is looking at the target and that at which the receiver is looking at it.

It was shown that the physical optics approximation used within the theorem is applicable if the bistatic angle is considerably less than $\pi$, but for angles greater than this, significant errors would occur. It should be noted that most of the data examined in this paper are for modest bistatic angles for which the theorem should apply. If this model applied for these targets then, on average, the monostatic and bistatic radar cross sections would be comparable. It is interesting to note that although such a relationship does not all the fit the data, the upper limit of the bistatic radar cross section is approximately equal to the mean monostatic radar cross section. The limited amount of data available makes this a tantalizing observation, to which unfortunately we currently give much weight because of the small amount of data. However if the mean and maximum bistatic radar cross sections follow different laws with respect to the monostatic this actually suggests that the proportional variance of the radar cross section increases with increasing target size, which seems unlikely.

It can be theorized that if large 'coherent' reflectors, such as flat plates and corner reflectors, do not contribute significantly to the radar cross section then the radar cross section might be proportional to the surface area of the vessel, i.e to the $2 / 3$ power of the volume. Reference [14] shows that this is indeed the case when the radar cross section is dominated by the smaller-scale features. The bistatic radar cross section is not affected by dihedral and trihedral reflectors as is the monostatic cross section. We might therefore expect the power law (the slope in the logarithmic relationship in equation 3 ) to be about 0.67 rather than the 'best fit' value of about 0.5 . The limited regression coefficient means, however, that the data which is available does not rule out the hypothesis that the law is actually a 'two-thirds' power law rather than a 'square root.' If the 'two-thirds' law is preferred, the 'intercept' would be at about $-7 \mathrm{dBm}^{2}$, rather than the value of $-3.27 \mathrm{dBm}^{2}$ given by the best fit.

On the other hand it may be noted that [10], equation 2, actually predicts the same 'square-root' law for the monostatic radar cross section, as is given by the best-fit for the bistatic radar cross section. Since, however, that equation (2) also seems to have been derived from a relatively small number of measurements, it may also be hypothesised that the power law in equation (2) should also actually be a two-thirds power.

It is probably unwise to trust the variation with frequency which was indicated in equation 4 . As well as the very low regression coefficient, the very steep slope is unlikely, and it might be noted that it is of the opposite slope from that reported in reference [10].

It should be noted that the overall pattern of radar cross section with angle will also contain 'flashes' at certain angles where flat plates act as mirrors to reflect the transmitted signal directly into the receiver, but the elevation angles of the transmitter in many cases will make it unlikely that this will be seen.

The fact that the data which is available seem to show no significant variation with geometry might at first sight seem surprising, but it should be remembered that the generic models of the monostatic radar cross sections of ships to not include an aspect-angle term. The lack of any discernable relationship with geometry may however also be due to the small number of measurements compared with the very wide range of possible bistatic geometries. 


\section{COMment On Bistaic Radar Cross Section OF OTHER TARGETS}

It should be noted that if equation (1) is applied to targets with a monostatic radar cross section of $0.5 \mathrm{~m}^{2}$ to $10 \mathrm{~m}^{2}$ $\left(-3 \mathrm{dBm}^{2}\right.$ to $\left.10 \mathrm{dBm}^{2}\right)$, more typical of other radar targets such as people, land vehicles or aircraft, it predicts bistatic radar cross section values in the range $-4.5 \mathrm{dBm}^{2}$ to $2 \mathrm{dBm}^{2}$ which is compatible with the common assumption that one assume that the bistatic radar cross section is very roughly an order of magnitude below the monostatic.

\section{CONCLUSIONS}

The most obvious conclusion from the analysis of the available data is that more data is needed, both to enhance the reliability of the data and to enable exploration of any possible systematic variation in radar cross section with bistatic angle and whether the relationship between monostatic and bistatic radar cross section should be expressed other than by a power law (a linear relationship in decibels). Key conclusions from the presented data are:

a) The mean bistatic radar cross section of a large target can be assumed to be the square root of the monostatic.

b) The 'intercept,' at which the monostatic and bistatic radar cross sections are expected to be the same is at a radar cross section of about $-6 \mathrm{dBm}^{2}$.

c) It is suspected that the true 'law' might be a two-thirds power law which is reported as a 'square root' law because of the limited amount of data available.

d) It seems that the maximum bistatic radar cross section might equal the mean monostatic cross section, but the data supporting this is very limited.

e) A variation of bistatic radar cross section with frequency has been observed but this is considered to be unreliable.

f) No relationship between radar cross section and bistatic geometry has been observed, but this may be due to the small number of measurements compared with the very wide range of possible bistatic geometries.

For large ships the reduction in bistatic target size is much more significant that any changes in sea clutter cross section [1] and reductions in 'spikiness' [2,3], so for the same 'sensitivity' (power density at the target, range to the receiver, receiver aperture, receiver sensitivity, dwell time etc.), the bistatic radar's sensitivity will be significantly less than that of the monostatic. This will have to be taken into account when considering the advantages of bistatic radar discussed in the introduction to this paper.

Since the measurements which have been analysed were made at frequencies between approximately $700 \mathrm{MHz}$ and $2.4 \mathrm{GHz}$ extrapolation to, for example, $\mathrm{X}$-band must be made with caution. There is accordingly a particular need for bistatic radar cross section measurements of ships at X-band.

\section{REFERENCES}

[1] W. A. Al-Ashwal, K. Woodbridge, and H. D. Griffiths, "Analysis of bistatic sea clutter Part I: Average reflectivity," IEEE Trans. Aerospace \& Electron. Sys., vol. 50, pp. 1283-1292, 2014.

[2] W. A. Al-Ashwal, K. Woodbridge, and H. D. Griffiths, "Analysis of bistatic sea clutter Part II: Amplitude statistics," IEEE Trans. Aerospace \& Electron. Sys, vol. 50, pp. 1293-1303, 2014.

[3] M. Ritchie, A. Stove, K. Woodbridge and H. Griffiths, "Monostatic and bistatic sea clutter texture and doppler spectra characterisation at Sband," IEEE Trans. Geoscience \& Remote Sensing, vol. 54, pp55335543, September 2016

[4] M. Cherniakov (Ed.) Bistatic Radar Principles and Practice, Chichester: Wiley, 2007, ISBN 978-0-470-02630-4

[5] H. D. Griffiths and C. J. Baker, An Introduction to Passive Radar, Norwood:Artech House, 2017, ISBN 978-1-630-81036-8

[6] A. G. Stove, M. S. Gashinova, S. Hristov and M. Cherniakov, M., "Passive maritime surveillance using satellite communication signals," IEEE Trans. Aerospace \& Electron. Sys. vol 53, pp 2987-2997, December 2017

[7] D. W. O’Hagan. A. Capria, D. Petri, V. Kubica, M. Greco F. Berizzi and A. G. Stove, "Passive bistatic radar (PBR) for harbour protection applications," proc. IEEE Radar Conf. 2012, pp 446-50.

[8] M. Inggs, G. Inggs, S. Sandenbergh, W. Al-Ashwal, K. Woodbridge and H. Griffiths, "Multistatic networked radar for sea clutter measurements," proc. IEEE Int. Geoscience \& Remote Sensing Symp. (IGARSS), 2011.

[9] M. I. Skolnik, Introduction to Radar Systems, 3rd Ed., New York: McGraw-Hill, 2001, ISBN 0-07-290980-3, Table 2.1.

[10] M. I. Skolnik, “An empirical formula for the radar cross section of ships at grazing incidence," IEEE Trans. Aerospace \& Electron. Sys. vol. 10, p292, March 1972.

[11] P. D. L. Williams, H. D. Cramp and K. Curtis, "Experimental study of the radar cross-section of maritime targets, IEE J. Electron. Circuits \& Sys. vol. 2, pp. 121-136. July 1978

[12] Skolnik, M. I. Introduction to Radar Systems 2nd. Ed. New York: McGraw-Hill,1980, ISBN 0-07-057909-1, section 2. 8

[13] J. W. Crispin, R. F. Goodrich, and K. M. Siegel, A Theoretical Method for the Calculation of Radar Cross Section of Aircraft and Missiles Report No. 2591-1-H, University of Michigan, 1959

[14] J. M. Williams, "Radar cross-section of large structures with complex microgeometry," IEE Proc. Vol. 137 pt F, pp. 221-228, August 1990. 